\title{
El m- learning, un nuevo escenario en la educación superior del Ecuador
}

\section{M-learning, a new scenario in higher education in Ecuador}

Gladys Gioconda Lagos

Universidad de Guayaquil, Ecuador

Universidad Agraria del Ecuador, Ecuador

Autor para correspondencia: gladys.lagos.r@hotmail.com

Fecha de recepción: 30 de agosto de 2018 - Fecha de aceptación: 15 octubre de 2018

Resumen: La tecnología, el internet y las telecomunicaciones han ido cambiando los esquemas comunes de resolver las cosas por formas innovadoras y creativas; con la tecnología móvil en auge, los nuevos modelos de smartphones al alcance de la mano, se convierte en un reto para el docente usar estos dispositivos como aliados en el proceso educativo. La presente investigación analiza la aplicación del aprendizaje móvil (M-learning) en la educación superior del Ecuador, en específico en la carrera de Sistemas Multimedia de la facultad de Filosofía de la universidad de Guayaquil donde se realizó un estudio no experimental descriptivo, con 200 estudiantes del I y II ciclo 2017-2018 y 20 docentes a quienes se les aplicó una encuesta estructurada de 25 preguntas con 17 ítems. Los resultados mostraron que el 96,2\% de los encuestados utilizan los dispositivos móviles para actividades académicas, el 83\% accede a contenidos digitales desde sus dispositivos móviles durante las clases; el 89\% consideran necesaria la aplicación de Mlearning en todas las asignaturas; el 78\% de los docentes encuestados, opinan, que fomenta el aprendizaje colaborativo entre los estudiantes, mejorando su rendimiento académico en un $90 \%$. Se concluyó que el Mlearning tiene un alto nivel de aceptación entre la comunidad universitaria, su uso ha logrado fomentar la comunicación y sobre todo crear en los estudiantes la cultura del aprendizaje móvil como una alternativa digital prometedora rumbo a la educación en línea, sin embargo, se hace necesaria la capacitación constante a los docentes y estudiantes sobre la inclusión del M-learning en el currículo, como un nuevo modelo de aprendizaje en la educación superior del Ecuador.

Palabras Claves: tecnología; aprendizaje; m-learning; telecomunicaciones; dispositivos móviles

\begin{abstract}
Technology, the Internet and telecommunications have been changing the common schemes of solving things by innovative and creative ways; With the booming mobile technology, the new models of smartphones at hand, it becomes a challenge for the teacher to use these devices as allies in the educational process. This research analyzes the application of mobile learning (M-learning) in higher education in Ecuador, specifically in the Multimedia Systems career of the Faculty of Philosophy at the University of Guayaquil where a non-experimental descriptive study was conducted, with 200 students of the I and II cycle 2017-2018 and 20 teachers to whom a structured questionnaire of 25 questions with 17 items was applied. The results showed that $96.2 \%$ of respondents use mobile devices for academic activities, $83 \%$ access digital content from their mobile devices during classes; $89 \%$ consider the application of M-learning necessary in all subjects; $78 \%$ of the teachers in attendance, think, that encourages collaborative learning among students, improving their academic performance by $90 \%$. It was concluded that M-learning has a high level of acceptance among the university community, its use has been able to promote communication and above all create in students the mobile learning culture as a digital alternative to online education, without However, there is a need for constant training for teachers and students on the inclusion of Mlearning in the curriculum, as a new learning model in higher education in Ecuador
\end{abstract}

Key Words: technology; learning; m-learning; telecommunications; mobile devices 


\section{Introducción}

\section{Tecnologías de la información y comunicación en la educación}

Las tecnologías de la información y comunicación (TIC), han transformado la forma de hacer las cosas en los diferentes ámbitos del ser humano, creando nuevas estrategias y modelos en el desarrollo individual o colectivo de la sociedad, siendo los medios ideales para romper las barreras geográficas, sociales y culturales, que permitan fomentar la igualdad de oportunidades, dinamizar el aparato productivo de los pueblos logrando desarrollar competencias necesarias para desenvolverse adecuadamente en la sociedad. Las TIC se han convertido en un catalizador Ávila (2011), para la consecución de oportunidades laborales, educativas, de entretenimiento, culturales y sociales que han contribuido a reducir la brecha digital aun presente en la sociedad.

El impacto de las tecnologías de la información y comunicación se han filtrado de una forma muy significativa, y su avance ha permitido innovar el proceso de enseñanza - aprendizaje refrescando las estrategias y desarrollando metodologías que permitan incluir las TIC en las aulas de clases Salinas (2004). Es importante señalar que las inclusiones de las TIC no garantizan el éxito del proceso educativo, si el docente no da un giro de innovación en la práctica pedagógica y busca adaptarse a las nuevas herramientas tecnológicas para lograr un aprendizaje significativo en los estudiantes Salvat (2005).

Las instituciones de educación superior han buscado mecanismos para integrar las nuevas tecnologías de información y comunicación en los procesos de formación, y para este proceso es necesario que los docentes actualicen sus conocimientos en el uso y manejo de la tecnología con la finalidad de desarrollar competencias y capacidades en la búsqueda de información en el análisis crítico y reflexivo en el trabajo colaborativo que contribuyan a la generación del conocimiento.

\section{Los dispositivos móviles en la educación}

Al hacer referencia al termino dispositivos móviles, se piensa en un celular, con infinidad de modelos y funcionabilidad que van desde precios como dios hasta excesivamente costoso, sin embrago también están en esta definición las tablet, los iPod entre otros, Fombona (2012). Los dispositivos móviles constituyen una de las tecnologías más usadas dando un acceso incomparable a la comunicación y la información.

El aprendizaje con dispositivos móviles, Mobile learning o m-learning (Traxler, 2005), se refiere al uso de pequeños dispositivos digitales portátiles tales como smartphones y tablets, estos aparatos permiten establecer la comunicación de datos e información de modo inalámbrico con rapidez y versatilidad. El aprendizaje móvil presenta beneficios como la accesibilidad, conectividad la ubicuidad es decir el acceso en cualquier momento y lugar, con lo que se rompen las barreras de tiempo y espacio. El m- learning constituye la versión mejorada del e-learning (aprendizaje en línea o virtual), misma que esta universalizada en todos los campos de acción del ser humano. El uso de tales dispositivos y sus aplicaciones implica adaptar materiales digitales con el fin de que sean mejor aprovechados. 
Hsu, Ching \& Snelson (2014) identifican como la principal prioridad de investigación en el campo del aprendizaje móvil, estrategias de enseñanza-aprendizaje, aprendizaje colaborativo, aprendizaje basado en juegos, aprendizaje basado en la investigación, simulación, difusión de contenidos enriquecidos y tutoría para un aprendizaje ubicuo.

A medida que aumentan la potencia, la funcionalidad y la asequibilidad de esos dispositivos, aumenta también su capacidad de apoyar el aprendizaje de maneras nuevas. Iniciativas innovadoras de aprendizaje móvil surgidas en todo el mundo han puesto de relieve ese potencial (Fritschi y Wolf, 2012b; Hylén, 2012; Isaacs, 2012b; Lugo y Schurmann, 2012; Roschelle, 2003; So, 2012; West, 2012b). Sin embargo, aún existe la resistencia docente a la incorporación del teléfono móvil como herramienta didáctica en sus clases, Para Parsons (2014) el concepto de movilidad para alcanzar un aprendizaje más fluido, adaptativo, colaborativo y exploratorio, promoviendo la porosidad entre la educación formal y la informal es fundamental.

\section{Métodos}

\section{Tipo de estudio}

El estudio llevado a cabo es de tipo no experimental descriptivo con un enfoque mixto, cuantitativo y cualitativo (León y Montero, 2004; Clares, 2006; Albert, 2007). La perspectiva metodológica utilizada se enmarca dentro del paradigma interpretativo que pretende la "comprensión de los fenómenos educativos a través del análisis de las percepciones e interpretaciones de los sujetos que intervienen en la acción educativa" (Colás y Buendía, 1998, p. 50).

\section{Población}

La población de estudio estuvo conformada por estudiantes $(n=200)$ y docentes $(n=20)$ que pertenecen al I y II ciclo del periodo lectivo 2017-2018 de sexto, séptimo y octavo semestre de la carrera de sistemas multimedia de la facultad de Filosofía de la Universidad de Guayaquil. La caracterización de la muestra se presenta en función de las variables socio académicas analizadas (Brazuelo, 2013): edad, género,

La media de edad de los estudiantes está comprendida entre los 22 y 40 años, la de los docentes se haya comprendida entre los 36 y 51 años (62,3\%). Por géneros, se tiene que el $42 \%$ son mujeres y el $58 \%$ son hombres, en el caso de los docentes los hombres superan a las mujeres $(52,9 \%$ y $47,1 \%$ respectivamente).

\section{Instrumentos}

Como instrumentos para la recolección y análisis de datos el cuestionario y la revisión documental, aplicado de forma presencial. El cuestionario ha sido previamente validado a través de la consulta a expertos y se ha aplicado a una prueba piloto obteniendo un alfa de Cronbach con valores de 0,582 (moderada) a 0.931 (muy alta) (Ruíz, 1995), Tabla 1. El instrumento constaba de 25 preguntas con 17 ítems y 8 dimensiones, de tipo dicotómicas, preguntas cerradas pluricotómicas con opciones múltiples jerarquizadas y abiertas, así mismo se le daba la opción a los encuestados de elegir si, no y otras con a escala de respuesta tipo Likert (1-4), con las que se 
buscó evaluar la la percepción general, grado de aceptación, las posibilidades educativas de los dispositivos móviles al hacer uso de m-learning como nuevo modelo pedagógico. Así como también varias de las preguntas hicieron referencia acerca de su preferencia entre una clase presencial o una que incluya a la al m-learning.

\section{Tabla 1 Dimensiones del cuestionario}

\begin{tabular}{|c|c|c|c|}
\hline Dimensiones & Ítems & $\begin{array}{l}\text { Alpha de } \\
\text { Cronbach }\end{array}$ & Magnitud \\
\hline Datos generales del teléfono móvil. & 1 & 0,912 & Muy alta \\
\hline $\begin{array}{l}\text { Posibilidades multimedia, ofimáticas, } \\
\text { conexión inalámbrica e Internet. }\end{array}$ & 4 & 0,753 & Alta \\
\hline Nivel de conocimientos previos en TIC & 1 & 0,933 & Muy alta \\
\hline $\begin{array}{l}\text { Ambientes y usos del teléfono móvil en el } \\
\text { centro educativo y en el aula. }\end{array}$ & 1 & 0,61 & Alta \\
\hline $\begin{array}{l}\text { Políticas educativas de prohibición de los } \\
\text { teléfonos móviles }\end{array}$ & 1 & 0,732 & Alta \\
\hline $\begin{array}{l}\text { Motivos y actitudes a favor del uso educativo } \\
\text { del teléfono móvil }\end{array}$ & 3 & 0,772 & Alta \\
\hline $\begin{array}{l}\text { Obstáculos y actitudes en contra de la } \\
\text { incorporación educativa del teléfono móvil }\end{array}$ & 2 & 0,672 & Alta \\
\hline $\begin{array}{l}\text { Valoración del teléfono móvil como } \\
\text { herramienta educativa }\end{array}$ & 4 & 0,876 & Muy alta \\
\hline
\end{tabular}




\section{Resultados}

Luego del estudio realizado, los resultados evidenciaron que los dispositivos móviles son altamente usados en el proceso de enseñanza aprendizaje, ya que el 96,2\% de los encuestados utiliza sus dispositivos móviles en sus horas de clases, al consultarle a los encuestados si acceden a contenidos digitales referentes a las asignaturas desde sus dispositivos móviles el 83\% contestó afirmativamente; el 89\% considera necesaria la aplicación de M-learning en todas las asignaturas.

También se les consulto a los estudiantes cuáles eran las principales razones por las cuales se debería incluir al m-learnig dentro del currículo de la carrera de sistemas multimedia a lo que el $33 \%$ contesto, que permite mantenerse informado de manera rápida y efectiva, el $25 \%$ piensa que fomenta el aprendizaje ubicuo lo cual va a eliminar las barreras de tiempo y espacio que en los estudiantes de la jornada nocturna es un grave problema por sus horarios laborales, también se obtuvo que un $18 \%$ y $16 \%$ sostienen que la aplicación del m-learning mejora la comunicación y fomenta el trabajo colaborativo mientas que un $8 \%$ considera que permite la flexibilización en el aprendizaje. Fig. 1.

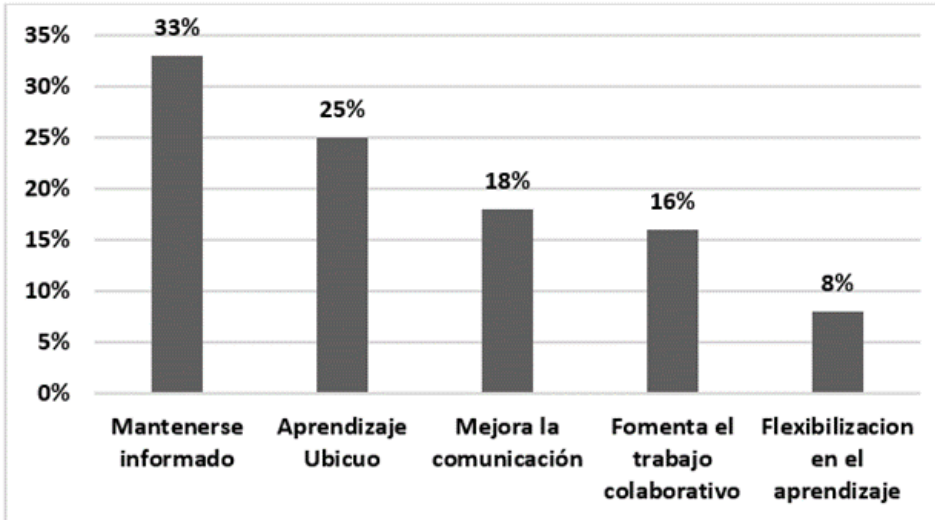

Figura 1. Razones para incluir al M-learning en la carrera de sistemas Multimedia

También se les consulto cuales son las principales actividades que realizan a través de sus dispositivos móviles, sigue liderando la mensajería instantánea con el $42 \%$ enviar y recibir mensaje, otro uso interesante del cual el docente es testigo ya que en las aulas de clases se evidencia la ausencia de cuadernos ya que con una foto se resuelve todo, el $20 \%$ lo usa como cuaderno virtual, medio de apuntes, igualmente el $20 \%$ en búsqueda de información y un $18 \%$ para compartir archivos tabla 1. De acuerdo a un estudio hecho, Baron (2016) se encontró que los estudiantes que usan aplicaciones móviles suelen recordar con mayor facilidad el patrón de búsqueda (habilidad procedural), que el resultado por sí mismo (habilidad cognitiva), es decir, el cerebro se adapta al uso de la tecnología, lo cual puede ser beneficioso en el trabajo bajo presión.

Tabla 1. Principales usos de los dispositivos móviles en clases

\begin{tabular}{lcr}
\hline & Frecuencia & Porcentaje \\
\hline Compartir archivos & 36 & $18 \%$ \\
Busqueda de información & 45 & $20 \%$ \\
Libreta de apuntes virtual & 42 & $20 \%$ \\
\hline
\end{tabular}




\begin{tabular}{lrr}
\hline Enviar y recibir mensajes & 77 & $42 \%$ \\
Total & 200 & $100 \%$ \\
\hline
\end{tabular}

Para el 78\% de los docentes encuestados, el M-learning fomenta el aprendizaje colaborativo entre los estudiantes, y su uso ha logrado mejorar el rendimiento académico de los estudiantes en un $90 \%$, debido que al poder usarlo como soporte en el proceso de enseñanza aprendizaje, los estudiantes pudieron enviar sus tareas a tiempo por ese medio así como también pudieron ser partícipes en los talleres de modo virtual a través del uso de video llamada por medio de la aplicación de WhatsApp instalada en la mayoría de los smartphones de los estudiantes. Para Elkheir y Mutalib (2015) el uso de los teléfonos móviles y tabletas podría fomentar que los alumnos se interesen en algún tema y en consecuencia que destinen más tiempo a su estudio. Al Hamdani (2013) señala que los dispositivos móviles funcionan como mediadores en el proceso de enseñanza y aprendizaje, ya que atravez de ellos se puede consultar diversos materiales educativos, de acuerdo a su estudio los participantes indicaron que los dispositivos les ayudaron a promover sus habilidades de pensamiento y a cooperar con sus pares; Igualmente para Fried (2008) los dispositivos móviles despiertan la motivación de los estudiantes dentro del aula de clase. 


\section{Conclusión}

El m-learning, aporta flexibilidad, accesibilidad, facilita la construcción del conocimiento, la resolución de problemas de aprendizaje y el desarrollo de destrezas o habilidades diversas de forma autónoma y ubicua, genera un fuerte motivación y dinamismo al proceso de enseñanza aprendizaje. La presencia de los dispositivos móviles en las aulas de clases es un hecho que va en aumento a escala mundial, y el docente no puede cerrar los ojos ante esta realidad.

A medida que los fabricantes desarrollan nuevas funcionabilidades a los dispositivos móviles estos adquieren mayor potencia, funcionabilidad y asequibilidad a todo tipo de información de forma casi inmediata, la resolución de problemas y la generación de conocimiento es parte del aprendizaje ubicuo. Por lo que se concluyó Se concluyó que el Mlearning tiene un alto nivel de aceptación entre la comunidad universitaria, su uso ha logrado fomentar la comunicación y sobre todo crear en los estudiantes la cultura del aprendizaje móvil como una alternativa digital prometedora rumbo a la educación en línea, sin embargo se hace necesaria la capacitación constante a los docentes y estudiantes sobre la inclusión y uso adecuado del M-learning en el currículo, como un nuevo modelo de aprendizaje en la educación superior del Ecuador.

\section{Agradecimientos}

Todo trabajo de investigación requiere tiempo esfuerzo y dedicación, por ello mi agradecimiento especial a Dios, sobre todo, a los estudiantes y a los compañeros docentes de la Carrera de Sistemas multimedia. 


\section{Bibliografía}

Albert, M.J. (2007). La investigación educativa. Madrid: McGraw Hill.

Ávila-Fajardo, G. P., \& Erazo, S. C. R. (2011). Propuesta para la medición del impacto de las TIC en la enseñanza universitaria. Education y educators, 14(1), 9.

Al Hamdani, D. (2015). Exploring students' learning style at a Gulf University: a contributing factor to effective instruction. Procedia-Social and Behavioral Sciences, 176, 124-128.

Baron, J., Hirani, S., \& Newman, S. (2016). Challenges in patient recruitment, implementation, and fidelity in a mobile telehealth study. Telemedicine and e-Health, 22(5), 400-409.

Brazuelo, F. (2013). El teléfono móvil: actitudes, usos y posibilidades educativas. Tesis doctoral. Universidad Nacional de Educación a Distancia. Madrid, España.

Elkheir, Z., \& Mutalib, A. A. (2015). Mobile Learning Applications Designing Concepts and Challenges: Survey. Research Journal of Applied Sciences, Engineering and Technology, 10(4), 438-442.

Clares, J. (2006). Informática Aplicada a la Investigación Educativa. Sevilla: Digital Atres.

Colás, M. y Buendía, L. (1998). Investigación Educativa. Sevilla: Alfar.

Fried, C. B. (2008). In-class laptop use and its effects on student learning. Computers \& Education, 50(3), 906-914.

Fritschi, J., \& Wolf, M. A. (2012). Mobile learning for teachers in North America exploring the potential of mobile technologies to support teachers and improve practive. Working paper series on mobile learning.

Fombona Cadavieco, Javier; Pascual Sevillano, María Ángeles; Madeira Ferreira Amador, María Filomena. Realidad aumentada, una evolución de las aplicaciones de los dispositivos móviles. Pixel-Bit. Revista de Medios y Educación, 2012, (41): 197-210, 2012.

Hsu, Y. C., Ching, Y. H., \& Snelson, C. (2014). Research priorities in mobile learning: An international Delphi study/Les priorités de recherche en matière d'apprentissage mobile: Une étude de Delphes internationale. Canadian Journal of Learning and Technology/La revue canadienne de l'apprentissage et de la technologie, 40(2).

Hylén, J., Damme, D. V., Mulder, F., \& D’Antoni, S. (2012). Open Educational Resources: Analysis of responses to the OECD country questionnaire.

Isaacs, S. (2012). Turning on mobile learning in Africa and the Middle East: Illustrative initiatives and policy implications. United Nations Educational, Scientific and Cultural Organization. 
León, O. y Montero, I. (2004). Métodos de investigación en Psicología y Educación. Madrid: McGraw-Hill.

Lugo, M. T., \& Schurmann, S. (2012). Turning on mobile learning in Latin America: Illustrative initiatives and policy implications. Paris, UNESCO. Retrieved on, 16(09), 2016.

Parsons, D. (2014). The future of mobile learning and implications for education and training. Increasing Access, 217.

Roschelle, J. (2003). Keynote paper: Unlocking the learning value of wireless mobile devices. Journal of computer assisted learning, 19(3), 260-272.

Ruíz, J. (1995). El Estudio de Casos. Fundamentos y Metodología. Madrid: UNED.

Salinas, J. (2004). Innovación docente y uso de las TIC en la enseñanza universitaria. International Journal of Educational Technology in Higher Education (ETHE), 1(1).

Salvat, B. G., \& Quiroz, J. S. (2005). La formación del profesorado como docente en los espacios virtuales de aprendizaje. Revista iberoamericana de educación, 36(1), 3.

So, H. J. (2012). Turning on mobile learning in Asia: Illustrative initiatives and policy implications. Policy focus: UNESCO working paper series on mobile learning, 1, 1-32.

Traxler, J. (2005, June). Defining mobile learning. In IADIS International Conference Mobile Learning (pp. 261-266).

West, D. (2012). How mobile devices are transforming healthcare. Issues in technology innovation, 18(1), 1-11. 